\title{
Effect of insoluble surfactants on a thermocapillary flow
}

Cite as: Phys. Fluids 33, 072106 (2021); https://doi.org/10.1063/5.0055373

Submitted: 28 April 2021 . Accepted: 16 June 2021 . Published Online: 14 July 2021

(D) Franco N. Piñan Basualdo, (D) R. Terrazas Mallea, (D) B. Scheid, (D) A. Bolopion, (D) M. Gauthier, and (D) P. Lambert 


\title{
Effect of insoluble surfactants on a thermocapillary flow
}

\author{
Cite as: Phys. Fluids 33, 072106 (2021); doi: 10.1063/5.0055373 \\ Submitted: 28 April 2021 - Accepted: 16 June 2021 . \\ Published Online: 14 July 2021
}

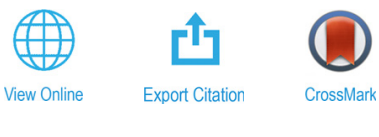

\author{
Franco N. Piñan Basualdo, ${ }^{1,2, a)}$ (D) R. Terrazas Mallea, ${ }^{3}$ (D) B. Scheid, ${ }^{1}$ (D) A. Bolopion, ${ }^{2}$ (D) M. Gauthier, ${ }^{2}$ (D) \\ and P. Lambert ${ }^{\top}$
}

\author{
AFFILIATIONS \\ ${ }^{7}$ Transfers, Interfaces and Processes (TIPs), Ecole Polytechnique de Bruxelles (CP 165/67), Université Libre de Bruxelles, \\ Avenue Franklin Roosevelt 50, 1050 Brussels, Belgium \\ ${ }^{2}$ FEMTO-ST Institute, Université Bourgogne Franche-Comté, CNRS, 24 rue Savary, F-25000 Besançon, France \\ ${ }^{3}$ Institute of Physical Chemistry, Polish Academy of Sciences, Kasprzaka 44/52, 01-224 Warsaw, Poland
}

${ }^{a)}$ Author to whom correspondence should be addressed: Franco.Pinan.Basualdo@ulb.be

\begin{abstract}
The thermocapillary effect, arising flow due to a temperature gradient along a fluid interface, is the dominant effect in some industrial and microfluidic processes and must be studied in order to optimize them. In this work, we analyze how insoluble surfactants adsorbed at the interface can affect such a flow. In particular, we analyze the case where the thermocapillary flow is induced at the air-water interface by locally heating it with an infrared laser, setup that is used to manipulate floating particles through the generated flow. Since water is a polar fluid, the air-water interface is easily polluted by surfactants. We developed a numerical model considering the uncontrolled presence of surfactants, which evidences that the effect of the surface contamination cannot be neglected, even for small surfactants concentration. The results of this numerical model were compared with different experimental measurements: particle tracking velocimetry, convection cell radius measurements, and thermography of the surface. All the experimental observations agree with the numerical model with the initial surface contamination being a fitting parameter. The model was then validated comparing its results with measurements for which a known quantity of surfactant was added to the interface. Finally, an analytical model was developed to explain the effects of the governing parameters, which agrees with the simulations and the experimental results. The developed models give us insight toward the miniaturization of the manipulation platform.
\end{abstract}

Published under an exclusive license by AIP Publishing. https://doi.org/10.1063/5.0055373

\section{INTRODUCTION}

Whenever a gradient of temperature exists along a fluid interface, an interfacial flow will arise due to the resulting surface stress. ${ }^{1}$ This effect, known as thermocapillary effect, has a strong impact on industrial processes, like welding ${ }^{2}$ and solidification. ${ }^{3}$ More recently, several applications have been developed in the field of microfluidics: a thermocapillary pump, ${ }^{4}$ droplets generation ${ }^{5}$ and migration, ${ }^{6,7}$ bubbles migration, ${ }^{8,9}$ and particles manipulation. ${ }^{10}$ These applications require understanding and controlling the thermocapillary flow. Experimentally, it can be difficult to obtain a perfectly clean fluid interface. Adsorbed impurities act like surfactants that rigidify the interface, ${ }^{11,12}$ effect known as surface elasticity, and can affect the thermocapillary flow. The presence of such an adsorbed layer of surfactants has been observed to cause symmetry breaking instabilities ${ }^{13,14}$ under some conditions. The stable regime has also been studied ${ }^{15}$ in a thin water layer under $2 \mathrm{D}$ configuration. Therefore, in this manuscript we close the gap and study the effect of an adsorbed layer of surfactants on the symmetric thermocapillary flow in a cylindrical configuration and a thick water layer.

In particular, we analyze the case where an infrared (IR) laser is used to generate a localized temperature increase at an air-water interface, inducing a thermocapillary flow. Such a configuration has been previously used to manipulate particles at the air-water interface. ${ }^{16,17}$ Water was selected as a fluid due to its high surface tension and low viscosity, giving place to high fluid velocities $(\approx 30 \mathrm{~mm} / \mathrm{s})$. However, since water is a polar fluid, impurities can easily be trapped at the interface, which makes it almost impossible to have a completely pure air-water interface. In Sec. II, we first introduce the theoretical frame and our particular problem. Then, in Sec. III, we present the developed numerical model and compare its results with three experimental measurements: particle tracking velocimetry (PTV), convection cell radius, and thermography measurements. Finally, in Sec. IV, we present the developed analytical model and compare its predictions with the simulation and experimental results. We find that no matter how small the 
amount of impurities, the induced surface elasticity always has an effect on the flow, similar to how a low fluid viscosity can completely change the flow profile compared to the inviscid case. The developed models help us to better understand the flow generation and give us insights toward the miniaturization of the thermocapillary manipulation platform.

\section{PHYSICAL CONSIDERATIONS}

In this section, we first introduce the theoretical frame of the work. Then, we present the particular problem we focus on, and we introduce the non-dimensional parameters governing the system.

\section{A. Theoretical frame}

Surface tension $(\sigma)$ can be defined as the required energy to increase the area of an interface per unit area $(\sigma=\partial E / \partial A)$. It is known that surface tension depends on the temperature and on the concentration of some species. ${ }^{18}$ In particular, some big molecules, known as surfactants, that have a hydrophobic tail and a hydrophilic head, adsorb at air-water or oil-water interfaces. They adsorb with their heads in the water phase and their tails in the other one, and reduce the surface tension. ${ }^{19}$ When a gradient of surface tension exists along the interface, whether it is due to a temperature or concentration gradient, a surface stress arises $\left(\tau_{\sigma}=\nabla_{S} \sigma\right)$. This stress is balanced by the viscous shear stress of each phase, resulting in

$$
\mu_{1} \frac{\partial \mathbf{U}_{1}}{\partial \mathbf{n}}-\mu_{2} \frac{\partial \mathbf{U}_{2}}{\partial \mathbf{n}}=\nabla_{S} \sigma,
$$

where $\mathbf{U}_{\mathbf{i}}$ and $\mu_{i}$ are the velocity vector and the dynamic viscosity of the fluid $i$, respectively, and $\mathbf{n}$ is the interface normal vector pointing from phase 1 to phase 2 . The operator $\nabla_{S}$ is the surface gradient operator $\left[\nabla_{S} \sigma=\nabla \sigma-\mathbf{n}(\mathbf{n} \cdot \nabla \sigma)\right]$. In the case of a liquid-gas interface, the gas' phase viscous stress can be neglected in (1). If we also assume that there is only one species of surfactant, we can write

$$
\mu \frac{\partial \mathbf{U}}{\partial \mathbf{n}}=-\left(\gamma_{T} \nabla_{S} T+\gamma_{\Gamma} \nabla_{S} \Gamma\right),
$$

where $T$ is the temperature, $\Gamma$ is the surface surfactant concentration, $\gamma_{T}=-\partial \sigma / \partial T$ and $\gamma_{\Gamma}=-\partial \sigma / \partial \Gamma$ are positive values hereby assumed constant. This last approximation is valid for low surfactant concentrations (for higher concentrations $\partial \sigma / \partial \Gamma=-E_{S} / \Gamma$ where $E_{S}$ if the surface elasticity ${ }^{12}$ ). It can be seen that, through (2), the momentum equation gets coupled with the energy and the surfactant transport equations at the interface. The surfactant transport equation can be complex for soluble surfactants, due to adsorption dynamics, or deformable interfaces, ${ }^{20}$ but in the case of an insoluble surfactant and a non-deformable interface, this equation is reduced to

$$
\frac{\partial \Gamma}{\partial t}+\nabla_{S} \cdot(\mathbf{U} \Gamma)=D_{S} \nabla_{S}^{2} \Gamma
$$

where $D_{S}$ is a surface diffusion coefficient.

\section{B. Particular problem}

In our case, ${ }^{16,17}$ we study the geometry shown in Fig. 1. A cylindrical volume of water whose top surface, in contact with air and where a layer of insoluble surfactants is adsorbed, is heated from above by an infrared laser beam pointed at the center of the water-air interface. Qualitatively, once the laser is turned on, the temperature at the

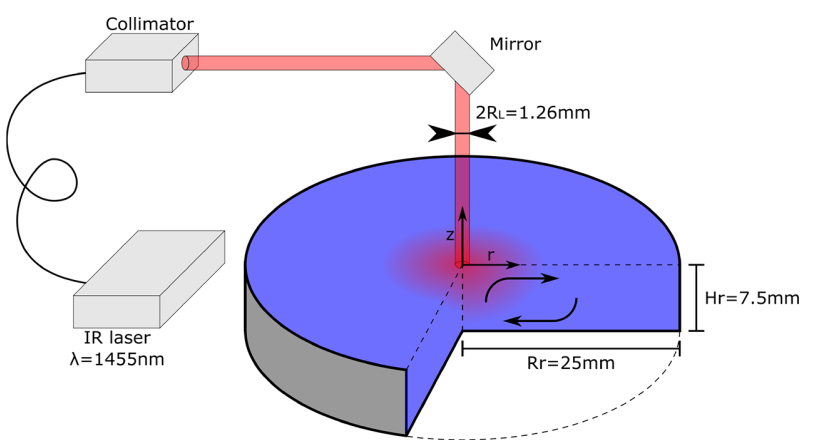

FIG. 1. Schematics of the system. An infrared laser (Keopsys Fiber Raman Laser CRFL-01-1455-OM1-B130-FA, wavelength $\lambda=1455 \mathrm{~nm}$, output power of up to $1 \mathrm{~W}$ ) is collimated and used to heat the air-water interface of a cylindrical volume of water (radius $R_{r}=25 \mathrm{~mm}$ and height $H_{r}=7.5 \mathrm{~mm}$ ) from above. The laser waist diameter is $2 R_{L}=1.26 \mathrm{~mm}$ (at the interface), and it is assumed to be constant (collimated beam).

center increases, creating a positive radial surface tension gradient, giving place to a positive radial flow. Because of mass conservation, a negative radial flow arises deep below the surface, generating a toroidal convection cell as shown by the arrows in Fig. 2(a) (Multimedia view). The radial flow at the free surface generates a depletion of surfactants around the laser spot, increasing the surface tension and counteracting the effect of the temperature increase.

In addition to the insolubility of the surfactants, we make further assumptions to simplify the modeling in the explored range of parameters (Table I): the interface deformations, the heat transfer to the air phase, and the buoyancy forces are neglected.

Indeed, surface deformations are considerable only for thin fluid layers. ${ }^{21,22}$ The vertical deformation of the interface in a similar configuration $^{23}$ has been predicted to be $\Delta H / R_{L}=\gamma_{T} \Delta T / \sigma_{0}$. If we consider $\Delta T \approx 2 \mathrm{~K}$ (the measured temperature increase for a laser power $P_{L}=40 \mathrm{~mW}$ ), we obtain $\Delta H / R_{L} \approx 0.004$, thus validating our assumption.

On the other hand, the heat transfer toward the air phase can be compared to the heat conduction in the water phase through the Biot number $B i=h R_{L} / \kappa$, where $h$ is the heat transfer coefficient to the air phase. Typical values for free convection of $\operatorname{air}^{24}$ are $h<25 \mathrm{~W} / \mathrm{m}^{2} \mathrm{~K}$, thus obtaining $B i<0.03$ and validating our assumption.

Finally, the strength of buoyancy forces can be compared to thermocapillary forces through the dynamic Bond number $B o_{d}=\rho \beta g R_{L}^{2} / \gamma_{T}=0.005$, where $\beta=200 \times 10^{-6} \mathrm{~K}^{-1}$ is the thermal expansion coefficient of water, and $g=9.8 \mathrm{~m} / \mathrm{s}^{2}$ is the gravitational acceleration.

In order to explain the absorption of the energy provided by the laser, we consider the light attenuation coefficient of water $\alpha$ $=3280 \mathrm{~m}^{-1}$ defined for the used wavelength $(\lambda=1455 \mathrm{~nm})$. Assuming a collimated Gaussian beam with a waist radius $R_{L}$, the absorbed volumetric power $\dot{q}_{L}$ (energy per unit volume and unit time) is $^{23}$

$$
\dot{q}_{L}=\frac{2 P_{L} \alpha}{\pi R_{L}^{2}} \exp \left(-2\left(\frac{r}{R_{L}}\right)^{2}+\alpha z\right),
$$

where $P_{L}$ is the laser power, $r$ the radial coordinate, and $z$ the vertical coordinate, with origin at the interface. Finally, (3) and (4) together 


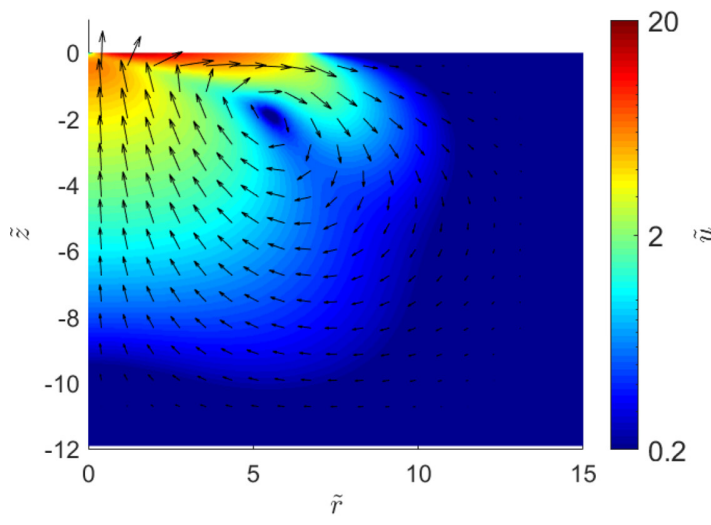

(a)

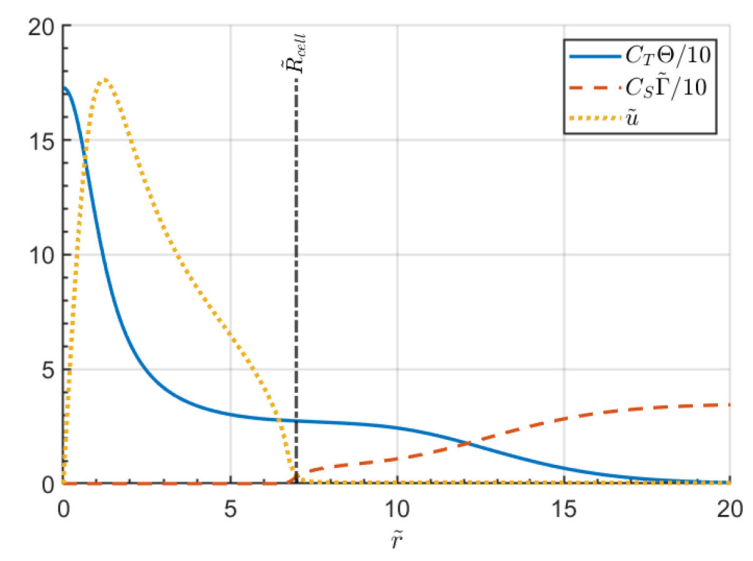

(b)

FIG. 2. Simulation results in the convective regime for $C_{T}=1 \times 10^{4}, C_{S}=30$, and $\tilde{t}=25$. (a) Non-dimensional velocity $\tilde{u}$ result. (b) Surface non-dimensional velocity $\tilde{u}$, temperature $C_{T} \Theta$, and surfactant concentration $C_{S} \tilde{\Gamma}$. The non-dimensional radius $\tilde{R}_{\text {cell }}=7$ is the point where the non-dimensional radial velocity vanishes. Multimedia views: https://doi.org/10.1063/5.0055373.1; https://doi.org/10.1063/5.0055373.2

with the mass, momentum (Navier-Stokes), and energy conservation complete the set of equations. Zero velocity, homogeneous temperature $\left(T=T_{0}\right)$, and homogeneous surfactant concentration $\left(\Gamma=\Gamma_{0}\right)$ are used as initial conditions. Regarding boundary conditions, we considered no slip, thermal insulation, and zero surfactant flux boundary conditions on the lateral recipient walls, no slip and constant temperature on the bottom wall, and zero vertical velocity (2), and thermal insulation at the air-water interface. For the detailed set of equations in cylindrical coordinates under the assumption of a flat interface, see the supplementary material.

These equations can be made non-dimensional by using $R_{L}$ as the characteristic length, $\tilde{t}=t \mu / \rho R_{L}^{2}$ as the non-dimensional time, $\tilde{\mathbf{U}}=\mathbf{U} \rho R_{L} / \mu$ as the non-dimensional velocity, $\tilde{P}=P \rho R_{L}^{2} / \mu^{2}$ as the non-dimensional pressure, $\Theta=\left(T-T_{0}\right) \kappa R_{L} / P_{L}$ as the nondimensional temperature, and $\tilde{\Gamma}=\Gamma / \Gamma_{0}$ as the non-dimensional surfactant concentration. The resulting set of equations is

TABLE I. Parameters' values used in this work.

\begin{tabular}{lcc}
\hline \hline Density & $\rho$ & $997 \mathrm{~kg} / \mathrm{m}^{3}$ \\
Dynamic viscosity & $\mu$ & $1.002 \mathrm{mPa} \mathrm{s}$ \\
Thermal conductivity & $\kappa$ & $0.607 \mathrm{~W} / \mathrm{m} \mathrm{K}$ \\
Specific heat capacity & $c_{p}$ & $4181 \mathrm{~J} / \mathrm{kg} \mathrm{K}$ \\
Surface tension $\left(T=20^{\circ} \mathrm{C}\right)$ & $\sigma_{0}$ & $72.9 \mathrm{mN} / \mathrm{m}$ \\
Thermocapillary coeff. & $\gamma_{T}$ & $155 \mu \mathrm{N} / \mathrm{m} \mathrm{K}$ \\
Laser wavelength & $\lambda$ & $1455 \mathrm{~nm}$ \\
Light attenuation coeff. & $\alpha$ & $3280 \mathrm{~m}^{-1}$ \\
Laser waist radius & $R_{L}$ & $630 \mu \mathrm{m}$ \\
Recipient radius & $R_{r}$ & $25 \mathrm{~mm}$ \\
Recipient height & $H_{r}$ & $7.5 \mathrm{~mm}$ \\
Laser power & $P_{L}$ & 20 to $200 \mathrm{~mW}$ \\
Surfactant surface pressure & $\gamma_{\Gamma} \Gamma_{0}$ & 0 to $150 \mu \mathrm{N} / \mathrm{m}$ \\
Surfactant diffusion coeff. & $D_{S}$ & $1 \times 10^{-7} \mathrm{~m}^{2} / \mathrm{s}$ \\
\hline
\end{tabular}

$$
\begin{gathered}
\tilde{\nabla} \cdot \tilde{\mathbf{U}}=0, \\
\frac{\partial \tilde{\mathbf{U}}}{\partial \tilde{t}}+\tilde{\mathbf{U}} \cdot \tilde{\nabla} \tilde{\mathbf{U}}=-\tilde{\nabla} \tilde{P}+\tilde{\nabla}^{2} \tilde{\mathbf{U}}, \\
\frac{\partial \Theta}{\partial \tilde{t}}+\tilde{\mathbf{U}} \cdot \tilde{\nabla} \Theta=\frac{1}{P r}\left(\tilde{\nabla}^{2} \Theta+\dot{\tilde{q}}_{L}\right),
\end{gathered}
$$

where $\dot{\tilde{q}}_{L}=2 \psi \exp \left(-2 \tilde{r}^{2}+\psi \tilde{z}\right) / \pi$ is the dimensionless absorbed volumetric power, $\psi=\alpha R_{L}$ describes the aspect ratio of the heated volume, and $\operatorname{Pr}=\mu c_{p} / \kappa$ is the Prandtl number. At the interface, (3) and (2) result

$$
\begin{gathered}
\frac{\partial \tilde{\Gamma}}{\partial \tilde{t}}+\tilde{\nabla}_{S} \cdot(\tilde{\mathbf{U}} C)=\frac{1}{S c} \tilde{\nabla}_{S}^{2} \tilde{\Gamma}, \\
\frac{\partial \tilde{\mathbf{U}}}{\partial \tilde{\mathbf{n}}}=-\left(C_{T} \tilde{\nabla}_{S} \Theta+C_{S} \tilde{\nabla}_{S} \tilde{\Gamma}\right),
\end{gathered}
$$

where $S c=\mu / \rho D_{S}$ is the surface Schmidt number, $C_{T}=\rho \gamma_{T} P_{L} / \mu^{2} \kappa$ is the thermocapillary number, and $C_{S}=\rho \gamma_{\Gamma} \Gamma_{0} R_{L} / \mu^{2}$ is the solutocapillary number. For the numerical values of these non-dimensional parameters, see Table II. For the detailed set of non-dimensional equations in cylindrical coordinates under the assumption of a flat interface, see the supplementary material.

\section{RESULTS}

In this section, we first present our numerical model and its results, observing three different regimes. Second, we compare the

TABLE II. Governing non-dimensional parameters and the values used in this work.

\begin{tabular}{llc}
\hline \hline Heated volume aspect ratio & $\psi=\alpha R_{L}$ & 2 \\
Prandtl number & $P r=\mu c_{p} / \kappa$ & 7 \\
Schmidt number & $S c=\mu / \rho D_{S}$ & 10 \\
Thermocapillary number & $C_{T}=\rho \gamma_{T} P_{L} / \mu^{2} \kappa$ & $5 \times 10^{3}$ to $5 \times 10^{4}$ \\
Solutocapillary number & $C_{S}=\rho \gamma_{\Gamma} \Gamma_{0} R_{L} / \mu^{2}$ & 20 to 80 \\
\hline
\end{tabular}


simulation results with three different experimental measurements (with an uncontrolled initial surfactant surface pressure): particle tracking velocimetry (PTV), convection cell radius, and thermography measurements. Finally, a controlled quantity of surfactants was added to the interface and the thermography measurements was repeated, validating our numerical model without any fitting parameter.

\section{A. Simulation results}

Finite elements, axisymmetric, time-dependent simulations were performed using Comsol multiphysics with the parameters shown in Table II, $\tilde{H}_{r}=10$ and $\tilde{R}_{r}=40$. For more details on the geometry discretization, see the supplementary material. The results have been observed to be independent of the recipient height $\tilde{H}_{r}$ and its radius $\tilde{R}_{r}$ provided they are larger than a minimum threshold value $\left(\tilde{H}_{r}>7.5\right.$, $\tilde{R}_{r}>30$, respectively) for the standard case $\left(C_{T}=1 \times 10^{4}\right.$ and $\left.C_{S}=30\right)$.

Depending on the values of $C_{S}$ and $C_{T}$, three regimes were observed: the convective, the transition, and the conductive regime.

If $C_{S}$ is small enough and $C_{T}$ large enough, we observe the convective regime. In this regime, a central area with no surfactant and a positive radial flow is obtained as shown in Fig. 2. Beyond a given radius, the surface tension gradients induced by temperature and concentration cancel each other and, according to (9), the shear stress at the surface vanishes. This radius coincides with $R_{\text {cell }}$, the radius at which the surface radial velocity vanishes [see the non-dimensional $\tilde{R}_{\text {cell }}=R_{\text {cell }} / R_{L}$ in Fig. 2(b) (Multimedia view)]. In addition, in this regime the maximum fluid velocity and temperature increase have been found to be independent of $C_{S}$. This is the regime we observe experimentally without added surfactant.

The transition regime is obtained for intermediate values of $C_{S}$ and $C_{T}$. In this regime, $\tilde{R}_{\text {cell }}$ approaches unity and the maximum fluid velocity and temperature were found to depend on $C_{S}$ (the first one decreases and the second one increases with $C_{S}$ ).

Finally, for large enough values of $C_{S}$ and small enough values of $C_{T}$, we obtain the conductive regime. In this regime, the effect of the temperature is not strong enough to create a complete depletion of surfactants at the laser spot. The stable state is a zero fluid velocity in the whole domain, since the surfactants completely counteract the temperature gradient. Therefore, the temperature profile is governed by heat conduction alone. We can find the limiting value for this regime as the point where the maximum temperature surface pressure is equal to the surfactant surface pressure: $C_{S \lim }=C_{T \Theta c}$ where $\Theta_{c}$ is the value of $\Theta$ at the center of the interface for a conduction only case. By performing a conduction only simulation to estimate $\Theta_{\mathcal{c}}$, we find $C_{\text {Slim }} \approx 0.2 C_{T}$.

\section{B. Experimental results}

The experimental validation of the above described numerical results was performed through three experimental observations (with an unknown surface contamination): particle tracking velocimetry (PTV), convection cell radius, and thermography measurements. All this measurements were performed in the convective regime. The PTV measurement allowed us observe the effect of contamination, but it could not be performed exactly at the interface. The convection cell radius measurement could be performed at the interface, but it does not allow us to observe the radial distribution. Finally, the thermography measurement allowed us to obtain the temperature distribution at the interface. Then, a controlled quantity of surfactant was added to the interface and the thermography measurements was repeated to validate our numerical model without any fitting parameter. However, with the added surfactant, the system was in the transient regime.

\section{PTV measurements}

To measure the flow velocity close to the air/water interface, the particle tracking velocimetry (PTV) method ${ }^{25,26}$ was used. A sketch of the setup is shown in Fig. 3(a). The optical system is composed of a green laser (MGL-III-532 $200 \mathrm{~mW}$ ), which is coupled to a fiber (Thorlabs P5-460B-PCAPC-1). Following the design methodology presented in Ref. 27, a set of convex and concave lenses was used to generate a thin collimated laser sheet, which was then used to illuminate the air-water interface. Polyamide seeding particles with a diameter of $50 \mu \mathrm{m}$ (Dantec Dynamics PSP-50) were submerged in the water

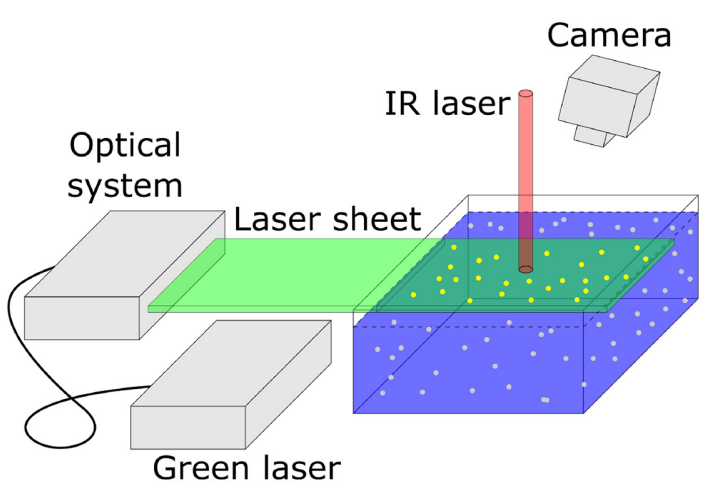

(a)

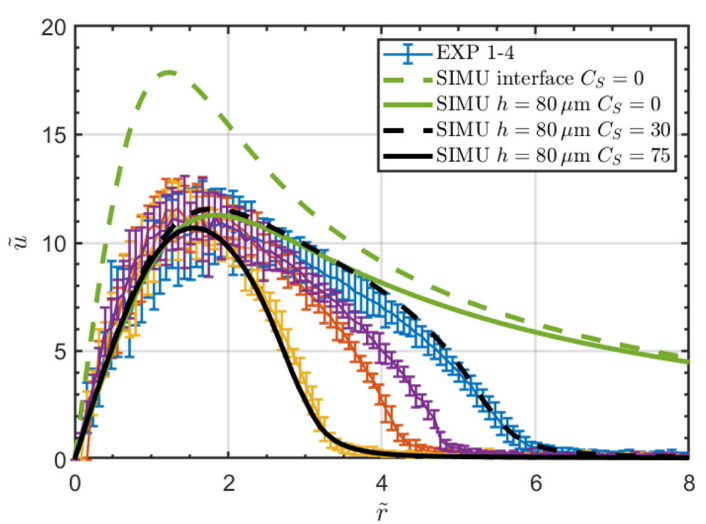

(b)

FIG. 3. PTV measurement. (a) Setup schematics. A green laser (MGL-III-532 $200 \mathrm{~mW}$ ) sheet is pointed toward the recipient. Only the illuminated tracers (50 $\mu \mathrm{m}$ diameter polyamide seeding particles) are seen by the camera. (b) Measurements and simulations for $C_{T}=1 \times 10^{4}$. The maximum velocity was used to fit the immersion depth at $h=80 \mu \mathrm{m}$. Then, the point at which the velocity vanishes was used to fit $C_{S}=30$ to 75 . 
bulk and stirred before each measurement. Because the seeding particles were only slightly denser than water $\left(\rho=1030 \mathrm{~kg} / \mathrm{m}^{3}\right)$, the convective flow was strong enough to move them along. The laser sheet was placed so that it would be as close as possible to the interface but ensuring that enough particles could be detected by the camera to reconstruct the flow velocity around the laser spot using the software presented in Ref. 28. As the exact experimental immersion depth of the laser sheet is unknown and due to its strong influence on the fluid velocity, it was decided to turn it into a fitting parameter. The immersion depth mainly affects the maximum value for the radial velocity, while $C_{S}$ mainly affects the point at which the velocity becomes zero. First, an immersion depth of $80 \mu \mathrm{m}$ (comparable with the size of the particles) was fitted using the maximum velocity value. Then, $C_{S}$ was fitted between 30 and 75 to rationalize the measurements. The process is shown in Fig. 3(b). The variation between each experimental measurement can be explained by a different initial surfactant concentration in each water sample. Our model considering an initial surface contamination succeeds in predicting a limited range of the laser effect, in contrast with the model that did not consider the surface contamination. Moreover, our model can explain the difference between measurements as the result of different contamination levels among different water samples.

\section{Convection cell radius at the surface}

To eliminate the immersion depth dependence, we decided to look directly at the interface. Some aluminum flakes (typical size $71 \mu \mathrm{m}$ ) were deposited on the water surface, where they float thanks to the surface tension. ${ }^{29}$ They are used as tracers to observe the surface flow since their inertia is low (Stokes number $S t<0.3$ ). Then, the laser was pointed toward a cluster of these floating flakes for at least $10 \mathrm{~s}$, where the generated thermocapillary flow repelled the flakes from the laser. The mean radius $R_{\text {cell }}$ of the area without flakes was estimated using the area entrapped by the dashed line shown in Fig. 4(a) (Multimedia view) where the central circle represents the laser spot. For the simulations results, since the surface velocity never becomes exactly zero, the non-dimensional radius $\tilde{R}_{\text {cell }}$ was defined as the point at which the non-dimensional radial velocity at the surface $\tilde{u}$ becomes lower than 0.3 . The obtained $\tilde{R}_{\text {cell }}$ value is not very sensitive to the threshold value in the range 0.3 to 1 , but quickly increases for lower values (for more details, see the supplementary material). The comparison between experimental measurements and simulations can be seen in Fig. 4(b) where the measurements agree with the results obtained with a fitting parameter $C_{S}=25$ (which is comparable to the lower value used to fit the PTV measurement $C_{S}=30$ ). In this case, we did not observe a considerable variation among different water samples.

\section{Thermography of the surface}

An experimental temperature measurement was obtained imaging the interface from above with an infrared camera Gobi-384. This eliminates both the immersion depth effect and the aluminum flakes distortions of the flow. On the other hand, thermography measurements at the fluid interface are not easy, ${ }^{30}$ since the expected temperature increase are quite small ( 2 and $8 \mathrm{~K}$ for 40 and $300 \mathrm{~mW}$ laser power, respectively). Such a technique has been successfully used to study the evaporation of sessile water droplets. ${ }^{31}$ A thermal image is taken before turning on the laser and another one $30 \mathrm{~s}$ after the laser has been turned on. The temperature map shown in Fig. 5(a) was obtained as the difference between both images. The temperature reading depends on both the camera sensor and ambient temperature, and these can change during the measurement. Therefore, sometimes it was necessary to add a constant offset $(<0.5 \mathrm{~K})$ to the temperature map to make the temperature increase vanish at large distances from the laser spot. Then, the point with the maximum temperature is defined as the laser center, and the temperature and distance from that center are computed for each pixel. The results are compared to the simulations' results as shown in Fig. 5(b), fitting $C_{S}$ to 60 . The same experiment was repeated for $C_{T}$ varying from $1 \times 10^{4}$ to $4 \times 10^{4}$, and the fitting parameter $C_{S}$ resulted always between 25 and 60. It is worth mentioning that while the convection cell radius strongly depends on $C_{S}$, in the conductive regime the maximum temperature does not.

\section{Controlled surfactants}

Finally, we added a controlled quantity of a known insoluble surfactants to the interface to compare simulations and experiments without any fitting parameter. Oleic acid (OA) was chosen as a surfactant

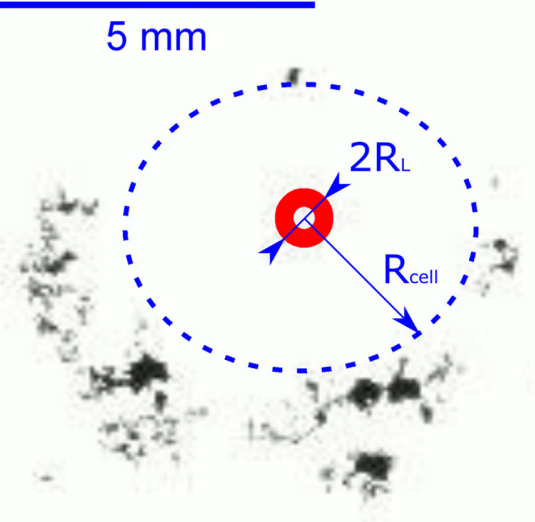

(a)

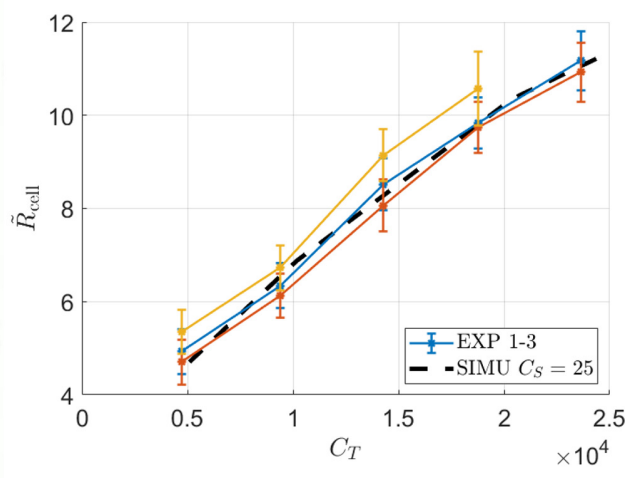

(b)
FIG. 4. Convection cell superficial radius measurement. (a) Measurement for $C_{T}=1 \times 10^{4}$. The dashed ellipse represents the convection cell and the red circle, the laser spot. (b) Measurements compared with simulations for different thermocapillary number $C_{T}$. Multimedia view: https://doi.org/10.1063/5.0055373.3. 


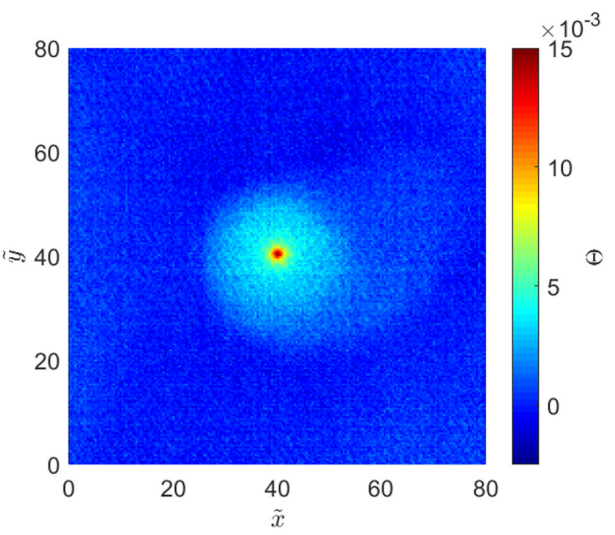

(a)

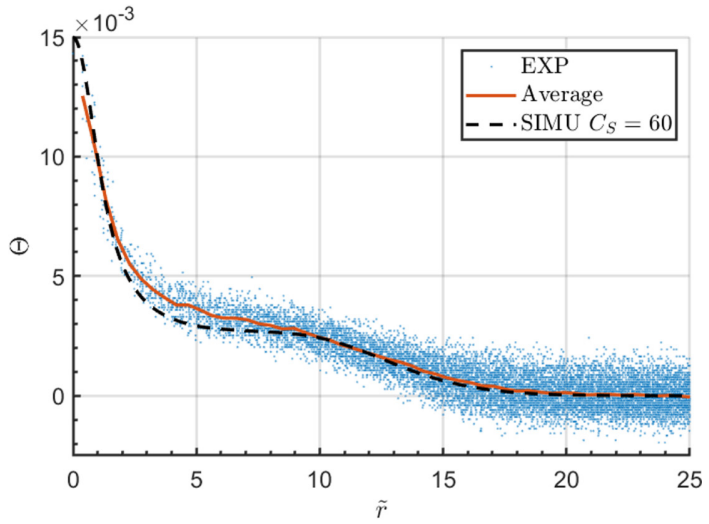

(b)

FIG. 5. Thermography measurement. (a) Difference between a first image taken before turning the laser on, and a second one $30 \mathrm{~s}$ after. (b) Comparison with simulations. The distance to the center and the temperature was computed for each pixel of a single measurement.

since it has been used in previous works. ${ }^{15}$ First, a Langmuir trough (MicroTrough XS, Kibron Inc.) was used to measure the change of surface tension as a function of the surface concentration of OA. The corresponding $C_{S}$ value as a function of the surface concentration is shown in Fig. 6. Two different regimes are clearly observed. For concentrations below a threshold value $\left(\Gamma_{e}=1.37\right.$ molecule $\left./ \mathrm{nm}^{2}\right)$, the variation of surface pressure with the OA concentration is very low. Meanwhile, for concentrations larger than $\Gamma_{e}$, the surface pressure quickly increases until reaching a saturation.

5 and $10 \mathrm{~nL}$ and of $\mathrm{OA}$, corresponding to a surface concentration $\Gamma_{a}=0.62$ molecule $/ \mathrm{nm}^{2}$ and $\Gamma_{b}=1.24$ molecule $/ \mathrm{nm}^{2} \quad\left(C_{S a}=220\right.$ and $\left.C_{S b}=440\right)$, respectively, were added to the interface. Smaller concentrations produce results that are hard to distinguish from those produced with no added surfactants, and at larger concentrations (above $\Gamma_{e}$ ), the surface pressure becomes large enough to almost stop

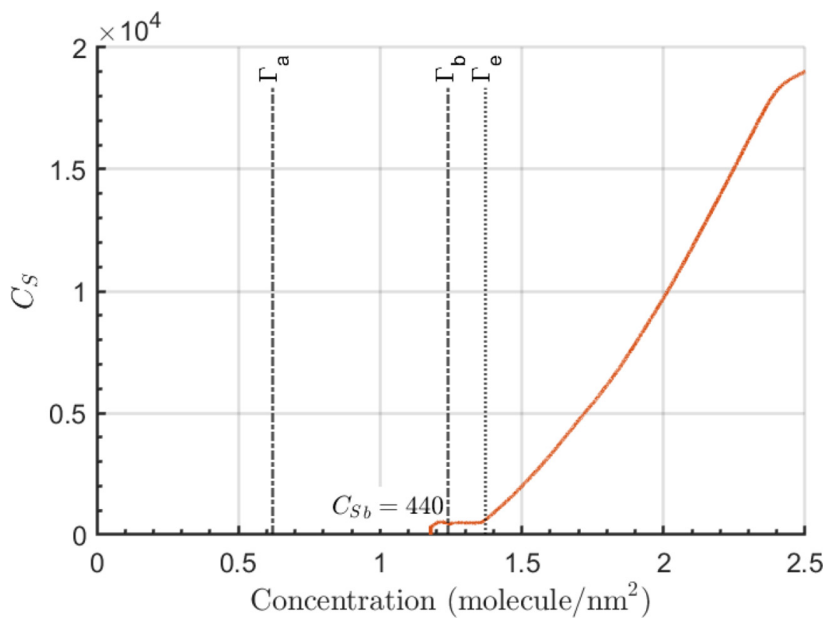

FIG. 6. Characterization of oleic acid $(\mathrm{OA})$ as surfactant. Corresponding $C_{S}$ value as a function of the surface concentration $\Gamma$. Isotherm measurements performed with a Langmuir trough with a compression rate of $2.04 \AA^{2} /($ min. molecule). Two regimes are observed with a transition concentration $\Gamma_{e}=1.37$ molecule $/ \mathrm{nm}^{2}$. the flow. These molecules, besides surface elasticity, could also induce surface shear and dilatational viscosity. ${ }^{32,33}$ In our configuration, like in many other experimental setups, the effect of surface elasticity and surface dilatational viscosities cannot be distinguished.

Then, the previously described procedure was followed to perform the thermography measurement. The results agree with the simulation using the corresponding $C_{S}$ value. When the effect of surfactant is much weaker than the thermocapillary flow, there is a good agreement with simulations as shown in Fig. 7(a). On the other hand, when the surfactant concentration becomes higher, there is an agreement with the simulations in the maximum temperature increase as shown in Fig. 7(b), but the plateau observed in the simulation is not observed in our experimental measurement. This can be due to second-order effects not accounted for in our model, like the induced surface viscosity, or the fact that the added surface surfactant concentration is close to $\Gamma_{e}$, from where the relationship between surface concentration and surface tension stops being linear as shown in Fig. 6. Moreover, we cannot guarantee that the initial surfactant concentration was homogeneous over the entire workspace.

We would like to mention that with the added OA, we observe some instabilities at higher laser powers $\left(C_{T}>4 \times 10^{4}\right)$. These instabilities can be explained by the competition between the thermocapillary and solutocapillary effects. ${ }^{13,14}$ We will not discuss these instabilities here since they are out of the scope of this article.

\section{DISCUSSION}

In this section, we present an analytical model we have developed to explain our simulations and experimental results. Similar configura-

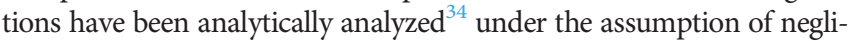
gible momentum and thermal convection could be neglected $(R e \ll 1$ and $P e_{T} \ll 1$ ), decoupling the temperature and momentum equations. In such a case, both the fluid velocity and the temperature increase turn out to be proportional to the laser power $\left(P_{L}\right)$. However, for the case of water and a convection dominated scenario like ours (local Péclet number $\operatorname{Pe}=\operatorname{Pr} \tilde{u} \approx 100$ ), no analytical model has been reported. Therefore, we developed a simple model using the concept of boundary layers as it was done in Ref. 35 for the solutocapillary 


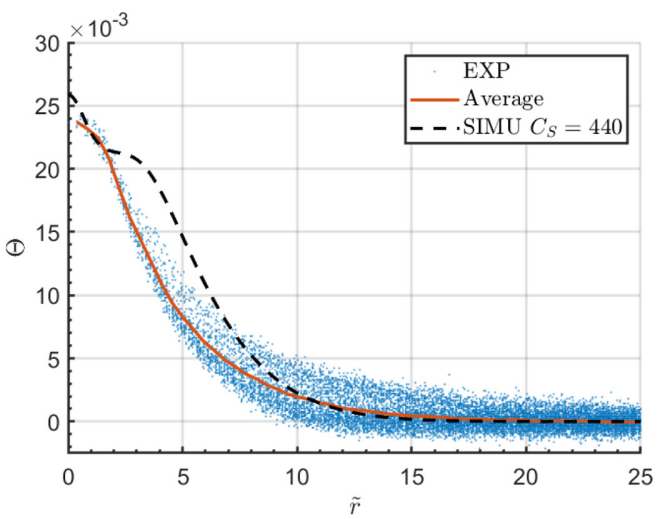

(a)

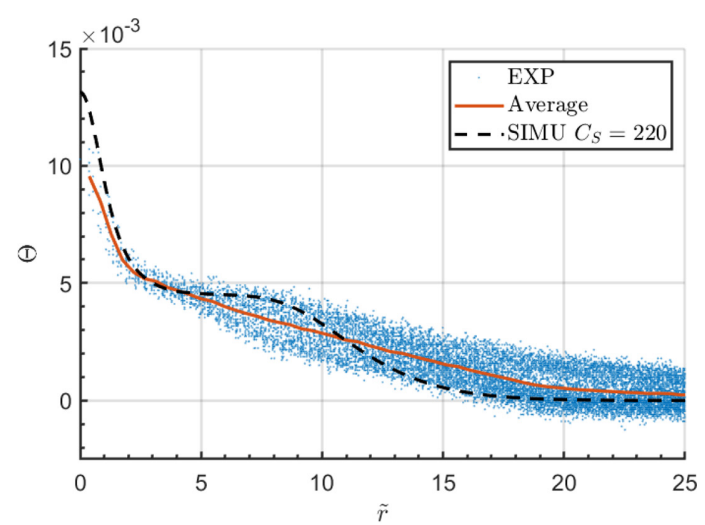

(b)

FIG. 7. Thermography results with added surfactants. (a) $O A$ concentration of $\Gamma_{a}=0.62$ molecule $/ \mathrm{nm}^{2}$ corresponding to $C_{S}=220$ and $C_{T}=4 \times 10^{4}$. (b) $O A$ concentration of $\Gamma_{b}=1.24$ molecule $/ \mathrm{nm}^{2}$ corresponding to $C_{S}=4400$ and $C_{T}=2 \times 10^{4}$.

effect. First, we analyze the clean interface case (without surfactant). We assume that away from the laser beam $(\tilde{r}>1)$ there are both a viscous and a thermal boundary layers whose thicknesses are, ${ }^{36}$ respectively,

$$
\begin{gathered}
\tilde{\delta}_{\mu} \propto \sqrt{\frac{\tilde{r}}{\tilde{u}_{S}}}, \\
\tilde{\delta}_{T}=\frac{\tilde{\delta}_{\mu}}{\operatorname{Pr}^{1 / 2}} \propto \sqrt{\frac{\tilde{r}}{\operatorname{Pr} \tilde{u}_{S}}},
\end{gathered}
$$

where $\tilde{u}_{S}$ is the non-dimensional radial fluid velocity and the subindex $S$ means at the interface. If we apply (7) to the interface for $\tilde{r}>1\left(\dot{\tilde{q}}_{L} \approx 0\right)$ under a steady-state condition and neglecting the conduction term in the radial direction, we obtain $\tilde{u}_{S} \partial \Theta / \partial \tilde{r}$ $=\operatorname{Pr}^{-1} \partial^{2} \Theta / \partial \tilde{z}^{2}$. If we approximate at the interface $\partial^{2} \Theta / \partial \tilde{z}^{2}$ $\propto-\Theta_{S} / \tilde{\delta}_{T}^{2}$, the result is

$$
\frac{\partial \Theta}{\partial \tilde{r}} \propto-\frac{\Theta_{S}}{\tilde{r}}
$$

From a surface stress balance point of view, we can approximate at the interface $\partial \tilde{u} / \tilde{z} \propto \tilde{u}_{S} / \tilde{\delta}_{\mu}$ and use (11) in (9) obtaining

$$
\Theta_{S} \propto \frac{\tilde{r}^{1 / 2} \tilde{u}_{S}^{3 / 2}}{C_{T}} .
$$

Then, since most of the energy brought by the laser will be evacuated through the thermal boundary layer by convection, that is, $P_{L} \propto r \delta_{\text {av }} \rho c_{p}\left(T_{S}-T_{0}\right) u_{S}$, where $\delta_{\text {av }}=P r^{-1 / 4} \delta_{\mu}$ is the geometric mean between $\delta_{\mu}$ and $\delta_{T}$. In non-dimensional form, this balance results

$$
1 \propto \tilde{r} \operatorname{Pr}^{3 / 4} \tilde{\delta}_{\mu} \Theta_{S} \tilde{u}_{S}
$$

Finally, combining (12) and (13) for $\tilde{r}=1$, and assuming $\tilde{u}_{\max }$ $\approx \tilde{u}_{S}(\tilde{r}=1)$ and $\Theta_{\max } \approx \Theta_{S}(\tilde{r}=1)$ we obtain

$$
\tilde{u}_{\max }=A C_{T}^{1 / 2} \operatorname{Pr}^{-3 / 8}
$$

$$
\Theta_{\max }=B C_{T}^{-1 / 4} \operatorname{Pr}^{-9 / 16} .
$$

We can fit the simulations results with $A=0.43$ and $B=0.54$ as shown in Figs. 8(a) and 8(b), respectively. Experimental temperature results also agree with both simulations and model results. As a comparison, in the case of a conduction dominated scenario, ${ }^{34}$ it was found that $\tilde{u}_{\max } \propto C_{T}$.

If now we consider the presence of surfactants, we assume that in the convective regime $\left(1 \ll \tilde{R}_{\text {cell }}\right)$, (14) and (15) are still valid. Under this assumption, we find $R_{\text {cell }}$ as the point at which the thermocapillary and solutocapillary effect cancel each other $\left(C_{T} \Theta_{S} \approx C_{S}\right)$. Therefore, we propose $\tilde{R}_{\text {cell }}=f\left(C_{T} \Theta_{\max } / C_{S}\right)$. If we assume a power law, we obtain

$$
\tilde{R}_{\text {cell }}=E\left(C_{T}^{3 / 4} C_{S}^{-1} \operatorname{Pr}^{-9 / 16}\right)^{\xi}
$$

The simulation results can be fairly fitted with $E=1.3$ and $\xi=2 / 3$ as shown in Fig. 8(c). The discrepancy between the model and simulations for $\tilde{R}_{\text {cell }}>20$ can be explained by the fact that we simulated a finite volume of water $\left(\tilde{R}_{r} \approx 40\right)$.

We simulated all the combinations of $\operatorname{Pr}=1,3,7$, and 14; $C_{T}=(5,10,20$, and 40$) \times 10^{3}$; and $C_{S}=10,20,40$, and 80 . When $\tilde{R}_{\text {cell }} \approx 1$ (approaching the transition regime), the velocity $\tilde{u}_{\max }$ becomes smaller than the value predicted by the model and $\Theta_{\max }$ becomes larger than the value predicted by the model.

\section{CONCLUSION}

In this manuscript, we analyzed the effect of an adsorbed layer of surfactants on a laser-induced thermocapillary flow. Qualitatively, an infrared laser is pointed toward a water volume from above, locally heating the air-water interface. The temperature gradient triggers an interfacial thermocapillary flow away from the laser spot. Due to the mass conservation, a flow toward the laser spot arises deep below the surface, generating a toroidal-like convection cell around the laser spot. The interfacial flow generates a depletion of surfactants around the laser spot, increasing the surface tension and counteracting the effect of the temperature. Analyzing the problem, we found four 


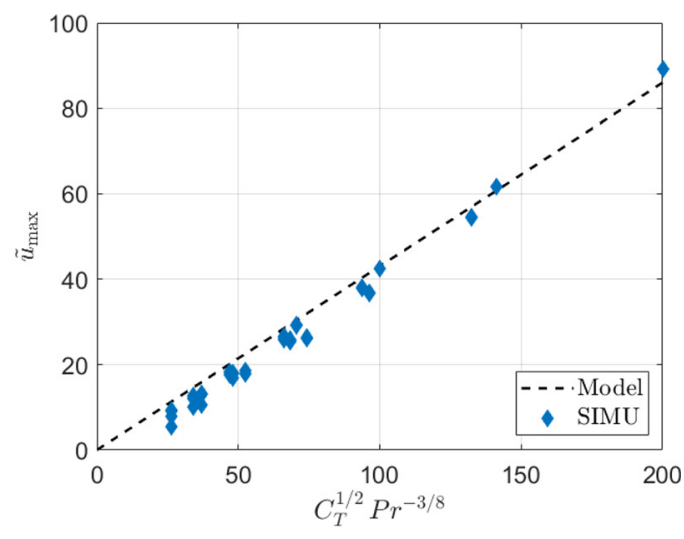

(a)

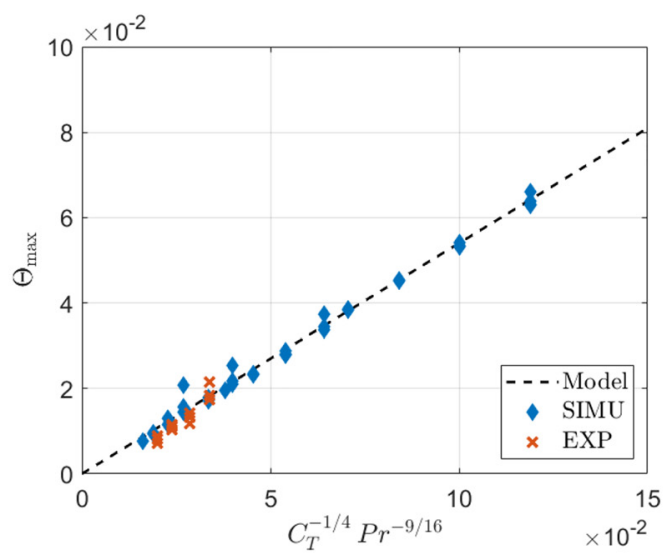

(b)

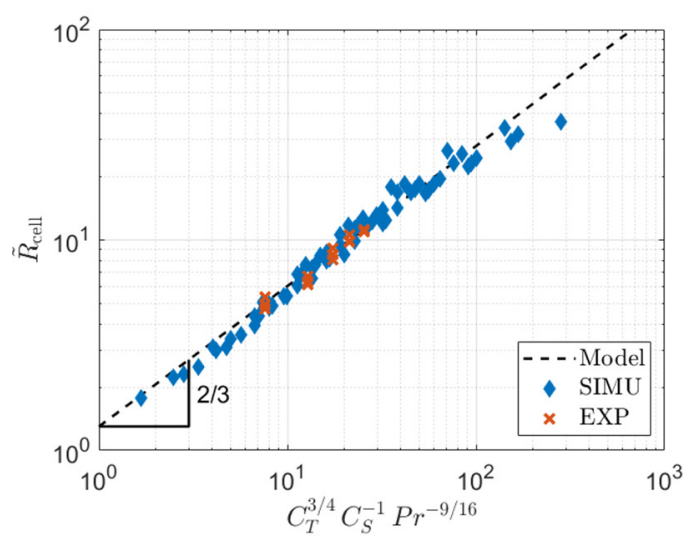

(c)

FIG. 8. Comparison between the simplified model (14), (15), and (16), the simulation results and experimental measurements. For the simulations results, we simulated all the combinations of $\operatorname{Pr}=1,3,7$, and $14, C_{T}=(5,10,20$, and 40$) \times 10^{3}$; and $C_{S}=10,20,40$, and 80 . (a) Maximum surface fluid velocity $\tilde{u}_{S}$. (b) Maximum surface temperature $\Theta_{S}$. (c) Convection cell radius $\tilde{R}_{\text {celll }}$. governing non-dimensional parameters (besides geometric ones): $\operatorname{Pr}=\mu c_{p} / \kappa$ is the Prandtl number, $S c=\mu / \rho D_{S}$ is the surface Schmidt number, $C_{T}=\rho \gamma_{T} P_{L} / \mu^{2} \kappa$ is the thermocapillary number (non-dimensional laser power), and $C_{S}=\rho \gamma_{\Gamma} \Gamma_{0} R_{L} / \mu^{2}$ is the solutocapillary number (non-dimensional surfactant concentration).

A numerical thermo-soluto capillary model was developed and applied to the problem described above. This model suggests that a (no matter how small) surfactant concentration affects the thermocapillary flow, reducing the size of the convection cell. Its results were compared with three different experimental observations (PTV, convection cell radius and thermography). The PTV and convection cell radius measurements are more sensitive to the surface contamination, but they necessarily modify the flow by introducing particles in it. On the other hand, the thermography measurement allowed us to obtain the temperature distribution at the interface, but this measurement is less sensitive to the surface contamination than the convection cell radius. First, we performed these measurements on different water samples with an uncontrolled surface contamination, obtaining good agreement with the same range for $C_{S}$ between 25 and 75 . Then, a known quantity of oleic acid was added as surfactant and the thermography measurements were repeated. The result was in good agreement with the simulations using the corresponding value for $C_{S}=220$ and $C_{S}=440$, thus validating the numerical model without fitting parameters.

Also, a simplified analytical model was developed for sufficiently low values of $C_{S}$. We observed a good agreement between this model and the numerical one in the explored range of parameters $\left(\mathrm{Pr}, \mathrm{C}_{T}\right.$, and $C_{S}$ ). We are confident that this simple model can be extended to other configurations as long as thermal convection dominates over conduction.

Such a system could be used to determine the level of contamination of an air-water interface. The convection cell radius measurement is very sensitive to the initial surface contamination level and could be used to characterize slightly contaminated interfaces where a simple surface tension measurement would not have enough resolution. On the other hand, the study of the flow gives us insights toward the miniaturization of the manipulation platform ${ }^{17}$ giving the parameters defining the range of the thermocapillary flow. For example, we see that by slightly increasing the surface concentration of surfactants, we could reduce the range of the thermocapillary flow without reducing its effect close to the laser spot.

\section{SUPPLEMENTARY MATERIAL}

See the supplementary material for the expanded governing equations in cylindrical coordinates, details on the geometry discretization, a discussion on the chosen threshold value to define the simulations cell radius, and an analysis of the effect of each governing non-dimensional parameter in the surface velocity and temperature.

\section{ACKNOWLEDGMENTS}

This work was funded by BELSPO (IAP 7/38 MicroMAST), FNRS grant (No. PDR T.0129.18), and the EUR EIPHI program (Contract No. ANR-17-EURE-0002). The authors thank the GRASP laboratory in Université de Liège (ULiège) for lending us the Langmuir trough. B.S. thanks the FRS-FNRS for financial support. 


\section{DATA AVAILABILITY}

The data that support the findings of this study are available from the corresponding author upon reasonable request.

\section{REFERENCES}

1'L. E. Scriven and C. V. Sternling, “The Marangoni effects," Nature 187, 186-188 (1960).

${ }^{2}$ K. Mills, B. Keene, R. Brooks, and A. Shirali, "Marangoni effects in welding," Philos. Trans. R. Soc. London, Ser. A 356, 911-925 (1998).

${ }^{3}$ F. Preisser, D. Schwabe, A. Scharmann et al., "Steady and oscillatory thermocapillary convection in liquid columns with free cylindrical surface," J. Fluid Mech. 126, 545-567 (1983).

${ }^{4}$ G. J. Amador, A. F. Tabak, Z. Ren, Y. Alapan, O. Yasa, and M. Sitti, "Thermocapillary-driven fluid flow within microchannels," arXiv:1802.00475 (2018).

${ }^{5}$ Y. Zhao, D. Wan, X. Chen, X. Chao, and H. Xu, "Uniform breaking of liquidjets by modulated laser heating," Phys. Fluids 33, 044115 (2021).

${ }^{6}$ Y. S. Ryazantsev, M. G. Velarde, R. G. Rubio, E. Guzmán, F. Ortega, and P. López, "Thermo-and soluto-capillarity: Passive and active drops," Adv. Colloid Interface Sci. 247, 52-80 (2017).

${ }^{7}$ K.-X. Hu, C.-Y. Yan, and Q.-S. Chen, "Instability of thermocapillary-buoyancy convection in droplet migration,” Phys. Fluids 31, 122101 (2019).

${ }^{8}$ M. A. Rahman, J. Cheng, Z. Wang, and A. T. Ohta, "Cooperative micromanipulation using the independent actuation of fifty microrobots in parallel," Sci. Rep. 7, 3278 (2017).

${ }^{9}$ M. Lu, J. Lu, Y. Zhang, and G. Tryggvason, "Numerical study of thermocapillary migration of a bubble in a channel with an obstruction," Phys. Fluids $\mathbf{3 1}$, 062101 (2019).

${ }^{10}$ K. Dietrich, N. Jaensson, I. Buttinoni, G. Volpe, and L. Isa, "Microscale Marangoni surfers," Phys. Rev. Lett. 125, 098001 (2020).

${ }^{11}$ E. Lucassen-Reynders, A. Cagna, and J. Lucassen, "Gibbs elasticity, surface dilational modulus and diffusional relaxation in nonionic surfactant monolayers," Colloids Surf. A 186, 63-72 (2001).

${ }^{12}$ L. Champougny, B. Scheid, F. Restagno, J. Vermant, and E. Rio, "Surfactantinduced rigidity of interfaces: A unified approach to free and dip-coated films," Soft Matter 11, 2758-2770 (2015).

${ }^{13}$ A. Mizev, A. Shmyrov, and A. Shmyrova, "On the shear-driven surfactant layer instability," arXiv:2101.02485 (2021).

${ }^{14}$ G. Koleski, A. Vilquin, J.-C. Loudet, T. Bickel, and B. Pouligny, "Azimuthal instability of the radial thermocapillary flow around a hot bead trapped at the water-air interface," Phys. Fluids 32, 092108 (2020).

${ }^{15}$ A. Shmyrov, A. Mizev, V. Demin, M. Petukhov, and D. Bratsun, "On the extent of surface stagnation produced jointly by insoluble surfactant and thermocapillary flow," Adv. Colloid Interface Sci. 255, 10-17 (2018).

${ }^{16} \mathrm{R}$. Terrazas Mallea, A. Bolopion, J. Beugnot, P. Lambert, and M. Gauthier, "Laser-induced thermocapillary convective flows: A new approach for noncontact actuation at microscale at the fluid/gas interface," IEEE/ASME Trans. Mechatronics 22, 693-704 (2017).
${ }^{17}$ F. N. Piñan Basualdo, A. Bolopion, M. Gauthier, and P. Lambert, “A microrobotic platform actuated by thermocapillary flows for manipulation at the airwater interface," Sci. Rob. 6, eabd3557 (2021).

${ }^{18}$ T. B. Nguyen and C. M. Phan, "Influence of hydrophilicity on the thermal-driven surfactant flow at the air/water surface," ACS Omega 3, 9060-9065 (2018).

${ }^{19}$ J. Eastoe and J. Dalton, "Dynamic surface tension and adsorption mechanisms of surfactants at the air-water interface," Adv. Colloid Interface Sci. 85, 103-144 (2000).

${ }^{20} \mathrm{H}$. A. Stone, "A simple derivation of the time-dependent convective-diffusion equation for surfactant transport along a deforming interface," Phys. Fluids A 2, 111-112 (1990).

${ }^{21}$ A. K. Sen and S. H. Davis, "Steady thermocapillary flows in two-dimensional slots," J. Fluid Mech. 121, 163-186 (1982).

${ }^{22}$ B. Messmer, T. Lemee, K. Ikebukuro, I. Ueno, and R. Narayanan, "Confined thermo-capillary flows in a double free-surface film with small Marangoni numbers," Int. J. Heat Mass Transfer 78, 1060-1067 (2014).

${ }^{23} \mathrm{H}$. Chraibi and J.-P. Delville, "Thermocapillary flows and interface deformations produced by localized laser heating in confined environment," Phys Fluids 24, 032102 (2012).

${ }^{24}$ P. Kosky, R. Balmer, W. Keat, and, and G. Wise, "Mechanical engineering," in Exploring Engineering, 5th ed., edited by P. Kosky, R. Balmer, W. Keat, and G. Wise (Academic Press, 2021), Chap. 14, pp. 317-340.

${ }^{25}$ T. Dracos, "Particle tracking velocimetry (PTV)," in Three-Dimensional Velocity and Vorticity Measuring and Image Analysis Techniques (Springer, 1996), pp. 155-160.

${ }^{26}$ S. Ferrari and L. Rossi, "Particle tracking velocimetry and accelerometry (PTVA) measurements applied to quasi-two-dimensional multi-scale flows," Exp. Fluids 44, 873-886 (2008).

${ }^{27}$ A. Prasad, "Particle image velocimetry," Curr. Sci. 79, 51-60 (2000), https:// www.jstor.org/stable/24103321.

${ }^{28}$ S. Dehaeck, P. Queekers, and P. Colinet, "Analyse par ptv du champ de vitesse de surface dans l'instabilité de Marangoni-Bénard," in Congrès Francophone de Techniques Laser (2010).

${ }^{29}$ D. Vella, "Floating versus sinking," Annu. Rev. Fluid Mech. 47, 115-135 (2015).

${ }^{30}$ R. G. Rubio, Y. S. Ryazantsev, V. M. Starov, G.-X. Huang, A. P. Chetverikov, P. Arena, A. A. Nepomnyashchy, A. Ferrus, and E. G. Morozov, "Thermography applied to interfacial phenomena, potentials and pitfalls," in Without Bounds: A Scientific Canvas of Nonlinearity and Complex Dynamics (Springer, 2013), pp. 157-182.

${ }^{31}$ F. Girard, M. Antoni, and K. Sefiane, "Infrared thermography investigation of an evaporating sessile water droplet on heated substrates," Langmuir 26, 4576-4580 (2010).

${ }^{32}$ J. Earnshaw, "Surface viscosity of water," Nature 292, 138-139 (1981).

${ }^{33}$ A. Choudhury, V. K. Paidi, S. K. Kalpathy, and H. N. Dixit, "Enhanced stability of free viscous films due to surface viscosity," Phys. Fluids 32, 082108 (2020).

${ }^{34} \mathrm{~T}$. Bickel, "Effect of surface-active contaminants on radial thermocapillary flows," Eur. Phys. J. E 42, 1-9 (2019).

${ }^{35}$ M. Roché, Z. Li, I. M. Griffiths, S. L. Roux, I. Cantat, A. Saint-Jalmes, and H. A. Stone, "Marangoni flow of soluble amphiphiles," Phys. Rev. Lett. 112, 208302 (2014).

${ }^{36}$ H. Schlichting and K. Gersten, Boundary-Layer Theory (Springer, 2016). 